\section{Inquérito de saúde em comunidades ribeirinhas do Amazonas, Brasil}

\author{
A health survey in riverine communities in \\ Amazonas State, Brazil
}

\section{Encuesta de salud en comunidades ribereñas del Amazonas, Brasil}

\section{Resumo}

Os inquéritos populacionais de saúde são instrumentos importantes para o reconhecimento de determinantes de morbidades, sobretudo em regiões de grande dispersão demográfica e baixa cobertura do sistema de saúde. O objetivo deste estudo foi apresentar os principais aspectos metodológicos e descrever as características socioeconômicas, demográficas e de saúde dos ribeirinhos de Coari, Amazonas, Brasil. Estudo transversal de base populacional conduzido com ribeirinhos residentes na zona rural do Município de Coari, no período de abril a julho de 2015. A amostra probabilística por conglomerados foi composta por 492 sujeitos. Os resultados indicaram que a maioria dos ribeirinhos é do sexo feminino (53\%), tem até 9 anos de estudos (68,5\%), apresenta em média renda familiar mensal equivalente a 1/3 do salário mínimo. Dentre os problemas de saúde relatados nos últimos 30 dias, destacaram-se as queixas álgicas $(45,2 \%)$. Os principais recursos utilizados nos cuidados com a saúde foram medicamentos alopáticos (70,3\%), superando o uso de plantas medicinais (44,3\%). Os ribeirinhos navegam em média 60,4km e demoram cerca de 4,2 horas para acessar a zona urbana do município. De maneira geral, a população ribeirinha estudada é caracterizada pelo baixo nível econômico e acesso limitado à zona urbana. Os problemas de saúde são solucionados na maior parte das vezes pelo uso de medicamentos alopáticos. As limitações geográficas que constituem barreiras ao acesso aos serviços de saúde e à melhoria das condições de vida dos ribeirinhos podem limitar a aquisição de informações epidemiológicas dessas populações.

Inquéritos Epidemiológicos; Nível de Saúde; População Rural
Abel Santiago Muri Gama 1

Tiótrefis Gomes Fernandes 2

Rosana Cristina Pereira Parente 3

Silvia Regina Secoli 4

doi: 10.1590/0102-311X00002817

\section{Correspondência}

A. S. M. Gama

Instituto de Saúde e Biotecnologia, Universidade Federal do Amazonas.

Estrada Coari-Mamiá 305, Coari, AM 69460-000, Brasil. abelsmg@hotmail.com

1 Instituto de Saúde e Biotecnologia, Universidade Federal do Amazonas, Coari, Brasil.

2 Faculdade de Educação Física e Fisioterapia, Universidade Federal do Amazonas, Manaus, Brasil.

3 Instituto Leônidas re Maria Deane, Fundação Oswaldo Cruz, Manaus, Brasil.

${ }_{4}$ Escola de Enfermagem, Universidade de São Paulo, São Paulo, Brasil. 


\section{Introdução}

Inquéritos populacionais de saúde constituem instrumentos importantes para o reconhecimento de determinantes de morbidades e desigualdades sociais 1 . São capazes de viabilizar a avaliação das políticas públicas previamente implantadas, sobretudo em grupos sociais excluídos ou pouco estudados em diferentes desfechos de saúde 1,2,3. No Brasil, são poucas as informações epidemiológicas sobre as populações ribeirinhas da Amazônia.

As populações ribeirinhas da Amazônia representam uma mistura de diferentes grupos sociais (indígenas, nordestinos e migrantes de outras regiões). Essas populações vivem em áreas rurais, às margens de rios e lagos na Amazônia brasileira, distribuindo-se em uma área geográfica com cerca de 5.020.000 km² 4,5. No Estado do Amazonas, a população estimada, no ano de 2010, foi de 3.483 .985 habitantes e $728.495(20,1 \%)$ viviam em área rural 6.

O cotidiano dos ribeirinhos às margens dos rios e sob influência das dinâmicas das águas (cheias e vazantes) impõe restrições de ordem multidimensional. A economia encontra-se alicerçada nas atividades de pesca e extrativismo vegetal. A cultura traz no seu bojo forte herança dos povos indígenas, com hábitos alimentares diferenciados, uso de plantas medicinais e agricultura de subsistência 4 . Os ribeirinhos, de modo geral, são destituídos de infraestrutura mínima de saneamento básico, energia elétrica e serviços de saúde 7 . A assistência à saúde é centralizada na zona urbana dos municípios, com ações esporádicas dos profissionais de saúde nas comunidades. $\mathrm{O}$ acesso à zona urbana costuma ser limitado pelas condições financeiras e, sobretudo, pela distância, que pode superar $500 \mathrm{~km}$ e deslocamento de vários dias, realizado somente por via fluvial.

Adicionalmente, o clima tropical e a floresta com dimensões colossais, atrelados à proximidade do homem com tal ambiente, constituem um fator singular para o desenvolvimento de agravos de saúde típicos da Região Amazônica. Investigações prévias apontam que fatores como baixa escolaridade, condição econômica insuficiente 7,8, ausência de saneamento básico 7,9,10, aliadas ao rol de doenças infecciosas endêmicas da região 11,12, parecem ter associação importante no perfil epidemiológico dessa população.

Nesse sentido, considerando a peculiaridade da região Amazônica, a dinâmica diferenciada no autocuidado da saúde da população ribeirinha e a necessidade de informações em saúde para o planejamento, o monitoramento e a gestão das intervenções 3, idealizou-se o estudo Saúde, Medicamentos e Automedicação em Ribeirinhos do Amazonas (SAMARA - Médio Solimões). Trata-se do primeiro estudo desenhado para conhecer as condições de vida e de saúde da população ribeirinha da região do Médio Solimões, Amazonas. Desse modo, o objetivo deste estudo é apresentar os principais aspectos metodológicos do SAMARA e descrever as características socioeconômicas, demográficas e de saúde dos ribeirinhos de Coari, Amazonas.

\section{Método}

\section{Delineamento}

SAMARA - Médio Solimões é um estudo transversal de base populacional conduzido com ribeirinhos residentes na zona rural do Município de Coari, realizado no período de abril a julho de 2015. O SAMARA avaliou aspectos socioeconômicos, condições de moradia e de acesso a serviços, condições de saúde, como uso de medicamentos, nutrição, atividade física, pressão arterial da população em geral e fragilidade entre os idosos.

\section{População de estudo}

O Município de Coari localiza-se na região central do Estado do Amazonas, na calha média do rio Solimões. Encontra-se a $363 \mathrm{~km}$ distante da capital Manaus. O acesso ao município é realizado com embarcações - via fluvial (9 a 30 horas de viagem) ou aérea (uma hora). A população local, no ano de 2010, era de 75.965 habitantes, sendo a 4a maior do Amazonas. A população urbana cresceu 20\% entre os anos de 2000 e 2010, enquanto se observou redução da rural (4,6\%). A despeito dessa redução, cerca 
de um terço dos habitantes de Coari viviam na zona rural - comunidades ribeirinhas dispersas ao longo das margens do rio Solimões, lagos e igarapés - distribuídos em uma área geográfica de $57.976 \mathrm{~km}^{2}$. A densidade demográfica era de 1,3 pessoas por $\mathrm{km}^{2} 6$.

Com recursos advindos da bacia extrativista de gás natural, Coari tem o maior Produto Interno Bruto (PIB) do interior do Estado do Amazonas 13. Os indicadores de saúde melhoraram nas últimas duas décadas. Apesar disso, os índices ainda são inferiores quando comparados aos indicadores do Estado do Amazonas e do Brasil (Tabela 1).

O sistema de saúde local é composto, atualmente, de um hospital de média complexidade com 105 leitos; 12 unidades básicas de saúde (UBS), sendo uma para atendimento das comunidades ribeirinhas; um Instituto Tropical de Medicina; um barco hospital para cobrir todas as comunidades ribeirinhas; um Laboratório Central de Análise Clínica; um Serviço de Emergência; um Núcleo de Vigilância Sanitária; uma Policlínica e um Centro de Atenção Psicossocial (CAPS) 14.

Os ribeirinhos vivem em comunidades, compostas por agrupamento de indivíduos, geralmente com laços familiares, com que compartilham costumes como hábitos alimentares e uso de produtos naturais. As casas são construídas em madeira (palafitas) na terra ou suspensas no rio (flutuantes). Não há padrão de ocupação do espaço e as comunidades diferem em relação à infraestrutura. Algumas possuem energia elétrica, telefonia móvel e antenas parabólicas, porém a maioria não dispõe de tais recursos nem de sistema de saneamento básico. Não há arruamento ou alinhamento das casas, e a distribuição espacial ocorre ao longo das margens dos rios, agrupadas ou consideravelmente distantes umas das outras 7.

Os ribeirinhos vivem da pesca, caça, agricultura de subsistência, especialmente do cultivo da mandioca para a produção de farinha (base alimentar) e do extrativismo da castanha e açaí, dentre outros produtos naturais obtidos na floresta e comercializados no município. Há dependência da população dos serviços e recursos da sede municipal, e o deslocamento das comunidades até a área urbana é feito por meio de canoas com motor (rabeta), o que, a depender da distância, tipo de transporte ou período do ano (seca ou cheia), pode demorar de minutos a três dias.

\section{Amostra}

A amostra foi estimada com base na população de referência registrada no cadastro no Sistema de Informação da Atenção Básica (SIAB) da Secretaria Municipal de Saúde (SEMSA) de Coari. Essa fonte foi adotada por conter registros mais precisos e atualizados das populações ribeirinhas. Para identificação das comunidades, considerou-se a distribuição das regiões utilizada pelos gestores locais de saúde.

Por se tratar de um inquérito em que vários aspectos foram investigados de modo simultâneo, adotou-se para o cálculo amostral a proporção de evento de 50\%, com precisão de 5\% e nível de $95 \%$ de confiança. Realizou-se ajuste para população finita e admitiram-se $20 \%$ de perda. A amostra calculada foi de 470 sujeitos, em 24 comunidades distribuídas (total de 135 comunidades cadastradas) em 8 regiões, entre lagos e rios da zona rural de Coari (Figura 1; Tabela 2).

Dentre as regiões ribeirinhas, a Alto Solimões é a mais populosa (3.501 habitantes), e a região de Codajás Mirim é a menos populosa (289 habitantes), com comunidades dispersas e poucas residências, uma espécie de canal de rio que liga as regiões do rios Copeá e Piorini e que em períodos de seca torna-se inexistente e confere isolamento da população (Tabela 2).

Foi realizada amostragem probabilística por conglomerados. O delineamento da amostra foi realizado em dois estágios: seleção aleatória da comunidade ribeirinha em cada região, com probabilidade proporcional à população da comunidade e seleção aleatória das residências. A seleção aleatória das residências foi realizada por meio de sorteio, de modo que ao chegar à comunidade, os domicílios foram selecionados de maneira alternada - o primeiro foi selecionado, excluindo o segundo, e entrevistando o seguinte, e assim por diante. Nos domicílios sorteados, todas as pessoas adultas foram entrevistadas.

Foram incluídos adultos ( $\geq 18$ anos) residentes nas comunidades ribeirinhas e que aceitaram participar do estudo. Dada a inviabilidade logística para retornar às comunidades, nos casos em que os domicílios sorteados encontravam-se sem moradores e na recusa em participar do estudo ou impossibilidade de participar, os entrevistadores seguiram para o domicílio seguinte. Dentre as comunidades 
Tabela 1

Comparação dos indicadores socioeconômicos e de saúde do Município de Coari, Estado do Amazonas, Brasil (1991, 2000 e 2010).

\begin{tabular}{|c|c|c|c|c|c|c|c|c|c|}
\hline \multirow[t]{2}{*}{ Indicadores } & \multicolumn{3}{|c|}{ Coari } & \multicolumn{3}{|c|}{ Amazonas } & \multicolumn{3}{|c|}{ Brasil } \\
\hline & 1991 & 2000 & 2010 & 1991 & 2000 & 2010 & 1991 & 2000 & 2010 \\
\hline População urbana [n (\%)] & $\begin{array}{l}21.081 \\
(54,50)\end{array}$ & $\begin{array}{l}39.504 \\
(58,90)\end{array}$ & $\begin{array}{l}49.651 \\
(65,40)\end{array}$ & $\begin{array}{l}1.502 .754 \\
(71,40)\end{array}$ & $\begin{array}{c}2.107 .222 \\
(74,90)\end{array}$ & $\begin{array}{c}2.755 .490 \\
(79,00)\end{array}$ & $\begin{array}{c}110.990 .990 \\
(75,60)\end{array}$ & $\begin{array}{c}137.953 .959 \\
(81,20)\end{array}$ & $\begin{array}{c}160.925 .792 \\
(84,40)\end{array}$ \\
\hline População rural [n (\%)] & $\begin{array}{l}17.597 \\
(45,50)\end{array}$ & $\begin{array}{l}27.592 \\
(41,10)\end{array}$ & $\begin{array}{l}26.314 \\
(34,60)\end{array}$ & $\begin{array}{l}600.489 \\
(28,60)\end{array}$ & $\begin{array}{l}705.335 \\
(25,10)\end{array}$ & $\begin{array}{c}728.495 \\
(21,00)\end{array}$ & $\begin{array}{c}35.834 .485 \\
(24,40)\end{array}$ & $\begin{array}{c}31.844 .926 \\
(18,80)\end{array}$ & $\begin{array}{c}29.830 .007 \\
(15,60)\end{array}$ \\
\hline População total [n (\%)] & $\begin{array}{l}38.678 \\
(100,0)\end{array}$ & $\begin{array}{c}67.096 \\
(100,00)\end{array}$ & $\begin{array}{c}75.965 \\
(100,00)\end{array}$ & $\begin{array}{c}2.103 .243 \\
(100,00)\end{array}$ & $\begin{array}{c}2.812 .557 \\
(100,00)\end{array}$ & $\begin{array}{c}3.483 .985 \\
(100,00)\end{array}$ & $\begin{array}{c}146.825 .475 \\
(100,00)\end{array}$ & $\begin{array}{c}169.798 .885 \\
(100,00)\end{array}$ & $\begin{array}{c}190.755 .799 \\
(100,00)\end{array}$ \\
\hline Taxa de mortalidade infantil * & 53,88 & 39,16 & 19,58 & 50,36 & 37,05 & 17,01 & 44,68 & 30,57 & 16,70 \\
\hline Expectativa de vida (anos) & 62,31 & 67,16 & 71,79 & 63,67 & 66,51 & 73,30 & 64,73 & 68,61 & 73,94 \\
\hline Taxa de fecundidade $* \star$ & 7,12 & 3,93 & 3,15 & 4,50 & 3,45 & 2,59 & 2,88 & 2,37 & 1,89 \\
\hline Taxa de analfabetismo *** & 45,80 & 31,52 & 17,02 & 23,82 & 15,50 & 9,84 & 20,07 & 13,63 & 9,61 \\
\hline $\begin{array}{l}\text { Renda familiar per capita } \\
\text { (Reais) \# }\end{array}$ & 146,23 & 159,29 & 347,20 & 345,82 & 351,63 & 539,80 & 447,56 & 592,46 & 793,87 \\
\hline IDH & 0,312 & 0,389 & 0,586 & 0,430 & 0,515 & 0,674 & 0,493 & 0,612 & 0,727 \\
\hline
\end{tabular}

IDH: Índice de Desenvolvimento Humano.

Fonte: Atlas do Desenvolvimento Humano no Brasil (http://www.atlasbrasil.org.br/2013/download/, acessado em 07/Jun/2016).

* Por 1.000 nascidos vivos;

** Número médio de filhos que uma mulher deverá ter ao terminar o período reprodutivo (15-49 anos de idade);

*** Em maiores de 15 anos;

\# Referente a 10 de agosto de 2010.

sorteadas no plano amostral, apenas quatro não foram visitadas. A inviabilidade de acesso ocorreu devido à distância (acima de 350km); dificuldades de navegabilidade em virtude da vegetação aquática, que bloqueava os canais de lagos; ou ausência dos moradores, por conta do êxodo para o Município de Coari, em decorrência das inundações. Dessa maneira, procedeu-se a novo sorteio para reposição.

\section{Abordagem da comunidade}

Para sensibilizar os ribeirinhos a participarem do estudo, utilizaram-se as seguintes estratégias: (1) comunicar os líderes comunitários e agentes comunitários de saúde (ACS) a respeito da importância da investigação e (2) divulgar o estudo por meio de atividade cultural denominada "Cine Ribeirinho". Os ACS ribeirinhos foram comunicados por meio de reuniões realizadas na SEMSA. Os líderes comunitários foram alertados previamente pelos ACS quanto ao estudo. O "Cine Ribeirinho" - cinema na comunidade - constituiu-se da reprodução de filmes do cinema mundial, durante o tempo em que a equipe de pesquisadores permaneceu nas comunidades e após as coletas. No intervalo dos filmes, eram exibidos vídeos de curta duração, cujos temas incluíam uso racional de medicamentos e prevenção de doenças.

\section{Questionário e coleta de dados}

Os sujeitos foram entrevistados em domicílio, utilizando-se um questionário padronizado com dez seções: (a) informações socioeconômicas e demográficas; (b) acesso aos serviços de saúde; (C) Alcohol Use Disorder Identification Test (AUDIT) adaptado para ribeirinhos 8; (D) hábitos tabagistas; (e) enfermidades autorrelatadas; (f) consumo de medicamentos; (g) Questionário Internacional de Atividade 

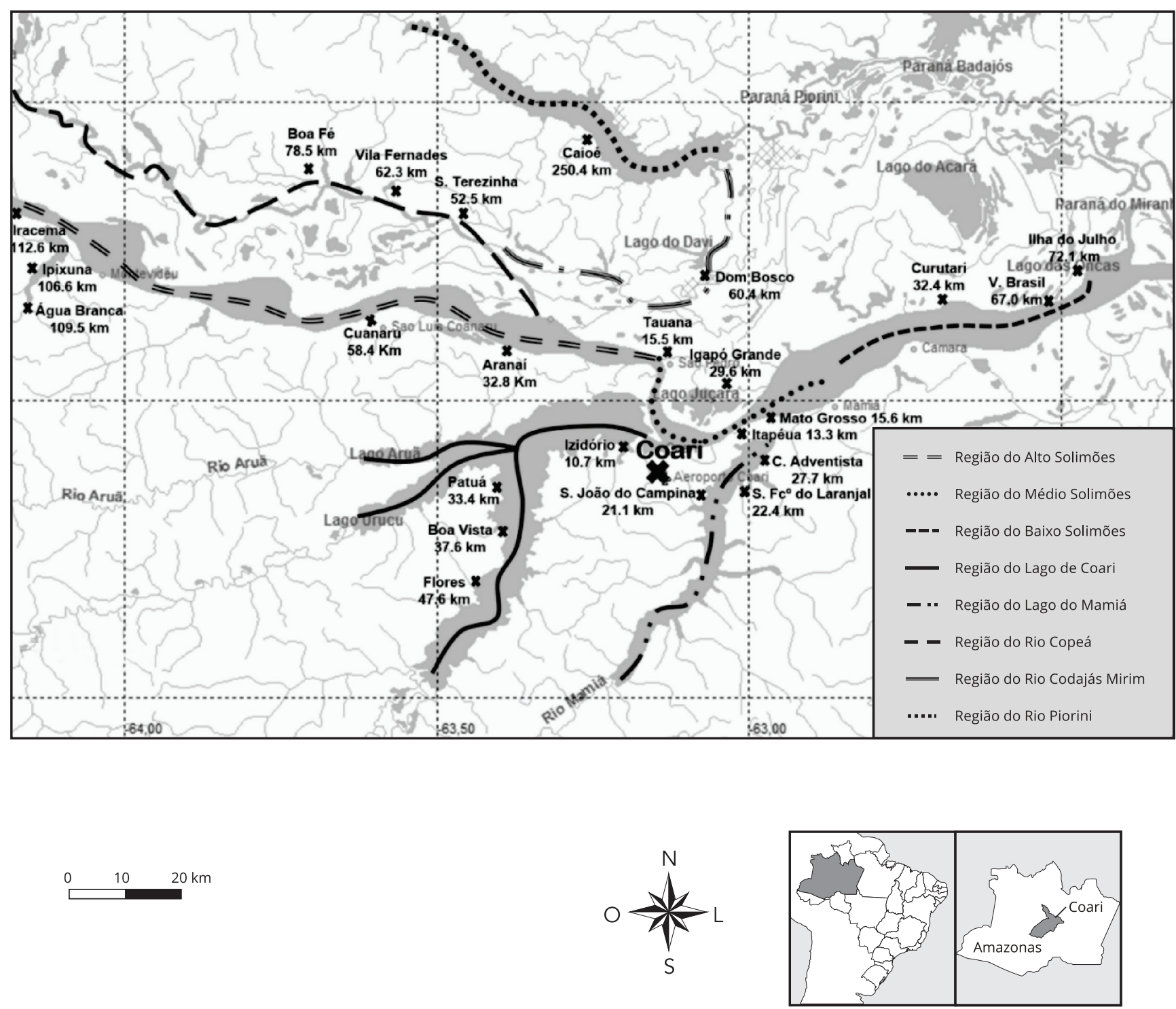

Física (IPAQ) 15,16; (h) inquérito sobre hábitos nutricionais; (i) screening para fragilidade em idosos 17; (j) dados antropométricos e de pressão arterial sistêmica (conforme protocolo da VI Diretriz Brasileira de Hipertensão) 18.

$\mathrm{Na}$ elaboração do questionário, considerou-se a terminologia usada pelos povos ribeirinhos, sobretudo no que tange às expressões empregadas para enfermidades e sinais e sintomas clínicos. Para essa finalidade, realizou-se teste piloto em duas comunidades ribeirinhas. A seção "enfermidades autorrelatadas", sofreu modificações que incluíram substituições de termos clássicos em saúde por expressões locais. Por exemplo, o termo "azia" que se refere à sensação de queimação que engloba a cavidade bucal até o estômago, foi substituído por "gastura”. 
Tabela 2

Descrição espacial das comunidades ribeirinhas segundo a população por região, distância, coordenadas das comunidades sorteadas e número de sujeitos na amostra por região e comunidade. Coari, Amazonas, Brasil, 2015.

\begin{tabular}{|c|c|c|c|c|c|}
\hline \multirow[t]{2}{*}{ Região/Comunidades } & \multirow{2}{*}{$\begin{array}{c}\text { População por região } \\
\text { e comunidades }\end{array}$} & \multirow{2}{*}{$\begin{array}{l}\text { Distância em relação } \\
\text { à zona urbana (km) }\end{array}$} & \multicolumn{2}{|c|}{ Coordenadas GPS } & \multirow{2}{*}{$\begin{array}{c}\text { Sujeitos } \\
\text { (n) }\end{array}$} \\
\hline & & & $\begin{array}{l}\text { Latitude } \\
\text { Sul }\end{array}$ & $\begin{array}{l}\text { Longitude } \\
\text { Oeste }\end{array}$ & \\
\hline Alto Solimões & 3.368 & & & & 103 \\
\hline Divino Espírito Santo Ipixuna & 221 & 106,6 & $03^{\circ} 51^{\prime} 40,0^{\prime \prime}$ & $63^{\circ} 52^{\prime} 57,4^{\prime \prime}$ & 24 \\
\hline Nossa Senhora do Cuanaru & 210 & 58,4 & $03^{\circ} 51^{\prime} 36,9^{\prime \prime}$ & $63^{\circ} 31^{\prime} 13,6^{\prime \prime}$ & 27 \\
\hline São Francisco do Aranaí & 75 & 32,8 & $03^{\circ} 55^{\prime} 43,1^{\prime \prime}$ & $63^{\circ} 17^{\prime} 56,6^{\prime \prime}$ & 10 \\
\hline Iracema & 199 & 112,6 & $03^{\circ} 48^{\prime} 36,7^{\prime \prime}$ & $63^{\circ} 59^{\prime} 05,7^{\prime \prime}$ & 26 \\
\hline São Sebastião da Água Branca & 138 & 109,5 & $03^{\circ} 53^{\prime} 07,9^{\prime \prime}$ & $63^{\circ} 52^{\prime} 30,1^{\prime \prime}$ & 16 \\
\hline Distância média (km) & & 84,0 & & & \\
\hline Médio Solimões & 2.568 & & & & 78 \\
\hline São Pedro do Tauana & 220 & 15,5 & $03^{\circ} 57^{\prime} 02,1^{\prime \prime}$ & $63^{\circ} 08^{\prime} 03,5^{\prime \prime}$ & 29 \\
\hline Nossa Senhora do Perpétuo Socorro de Itapéua & 280 & 13,3 & $04^{\circ} 03^{\prime} 31,8^{\prime \prime}$ & $63^{\circ} 01^{\prime} 42,1^{\prime \prime}$ & 24 \\
\hline São José do Mato Grosso & 101 & 15,6 & $03^{\circ} 59^{\prime} 08,6^{\prime \prime}$ & $63^{\circ} 02^{\prime} 11,0^{\prime \prime}$ & 9 \\
\hline Santa Maria do Igapó Grande & 182 & 29,6 & $04^{\circ} 02^{\prime} 11,5^{\prime \prime}$ & $63^{\circ} 01^{\prime} 02,7^{\prime \prime}$ & 16 \\
\hline Distância média (km) & & 18,5 & & & \\
\hline Baixo Solimões & 2.226 & & & & 68 \\
\hline Menino Deus Ilha do Julho & 107 & 72,1 & $03^{\circ} 52^{\prime} 25,3^{\prime \prime}$ & $62^{\circ} 38^{\prime} 34,1^{\prime \prime}$ & 17 \\
\hline Santo Antônio do Curutari & 148 & 32,4 & $03^{\circ} 55^{\prime} 10,5^{\prime \prime}$ & $62^{\circ} 55^{\prime} 27,7^{\prime \prime}$ & 25 \\
\hline Vila Brasil & 171 & 67,0 & $03^{\circ} 51^{\prime} 28,0^{\prime \prime}$ & $62^{\circ} 36^{\prime} 53,2^{\prime \prime}$ & 26 \\
\hline Distância média (km) & & 57,2 & & & \\
\hline Lago do Mamiá & 1.867 & & & & 57 \\
\hline Colônia Adventista & 153 & 27,7 & $04^{\circ} 02^{\prime} 18,9^{\prime \prime}$ & $62^{\circ} 55^{\prime} 02,3^{\prime \prime}$ & 22 \\
\hline São Francisco do Laranjal & 186 & 22,4 & $04^{\circ} 04^{\prime} 48,3^{\prime \prime}$ & $62^{\circ} 57^{\prime} 48,9^{\prime \prime}$ & 24 \\
\hline São João do Campina & 96 & 21,1 & $04^{\circ} 05^{\prime} 17,2^{\prime \prime}$ & $63^{\circ} 00^{\prime} 39,1^{\prime \prime}$ & 14 \\
\hline Distância média (km) & & 23,7 & & & \\
\hline Rio Copeá & 2.083 & & & & 64 \\
\hline Nossa Senhora do Perpétuo Socorro da Boa Fé & 222 & 78,5 & $03^{\circ} 40^{\prime} 11,7^{\prime \prime}$ & $63^{\circ} 34^{\prime} 53,6^{\prime \prime}$ & 28 \\
\hline Santa Terezinha & 99 & 52,5 & $03^{\circ} 45^{\prime} 49,6^{\prime \prime}$ & $63^{\circ} 23^{\prime} 59,9^{\prime \prime}$ & 13 \\
\hline Vila Fernandes & 187 & 62,3 & $03^{\circ} 42^{\prime} 52,0^{\prime \prime}$ & $63^{\circ} 28^{\prime} 14,3^{\prime \prime}$ & 27 \\
\hline Distância média (km) & & 64,4 & & & \\
\hline Lago de Coari & 2.431 & & & & 74 \\
\hline São Sebastião das Flores & 163 & 47,6 & $04^{\circ} 16^{\prime} 56,9^{\prime \prime}$ & $63^{\circ} 23^{\prime} 46,2^{\prime \prime}$ & 18 \\
\hline São José da Boa Vista & 99 & 37,6 & $04^{\circ} 12^{\prime} 49,5^{\prime \prime}$ & $63^{\circ} 23^{\prime} 43,8^{\prime \prime}$ & 15 \\
\hline São Tomé do Patuá & 114 & 33,4 & $04^{\circ} 09^{\prime} 26,3^{\prime \prime}$ & $63^{\circ} 23^{\prime} 57,1^{\prime \prime}$ & 14 \\
\hline Divino Espírito Santo do Izidório & 422 & 10,7 & $04^{\circ} 06^{\prime} 08,9^{\prime \prime}$ & $63^{\circ} 12^{\prime} 13,3^{\prime \prime}$ & 31 \\
\hline Distância média (km) & & 32,3 & & & \\
\hline Codajás Mirim & 289 & & & & 9 \\
\hline Dom Bosco & 125 & 60,4 & $03^{\circ} 50^{\prime} 24,7^{\prime \prime}$ & $63^{\circ} 06^{\prime} 32,7^{\prime \prime}$ & 9 \\
\hline Distância média (km) & & 60,4 & & & \\
\hline Rio Piorini & 570 & & & & 17 \\
\hline Nossa Senhora de Fátima do Caioé & 122 & 250,4 & $03^{\circ} 38^{\prime} 11,8^{\prime \prime}$ & $63^{\circ} 12^{\prime} 04,5^{\prime \prime}$ & 17 \\
\hline Distância média (km) & & 250,4 & & & \\
\hline Total & & & & & 470 \\
\hline
\end{tabular}

Participaram da coleta de dados professores e estudantes do Instituto de Saúde e Biotecnologia da Universidade Federal do Amazonas (ISB/UFAM). Realizou-se treinamento prévio da equipe. Os entrevistadores, que totalizaram 10, foram divididos em duplas.

As variáveis analisadas neste estudo foram as seguintes: seção (A) - sexo, faixa etária em anos (18-39; 40-59; 60 ou mais), raça (parda, branca, preta, amarela e indígena), religião (católico, evangélico ou outras), situação conjugal (vive só ou acompanhado), escolaridade em anos (analfabeto; 1-4; 5-9 
e 10 ou mais), atividade laboral (não exerce atividade laboral, agricultura e pesca, funcionário público, outros), renda familiar mensal per capita em Reais (10-85; 86-140; 141-240; 241 ou mais), número de moradores no domicílio (até 5; 6 ou mais); seção (B) - distância de acesso à zona urbana (menos de $50 \mathrm{~km}$; de 50-100km; mais de $100 \mathrm{~km}$ ), tempo para acessar a zona urbana (menos de 1 hora; de 1-4 horas; mais de 4 horas), frequência de idas à zona urbana (mais de uma vez por mês, apenas uma vez por mês, não costumo ir à cidade), procura pelos serviços de saúde nos últimos 30 dias (sim ou não); seção (C) - uso de bebida alcoólica (sim ou não); seção (D) - fuma (sim ou não); seção (E) - problema de saúde nos últimos 30 dias (sim ou não), tipo de problema de saúde referido nos últimos 30 dias (a distribuição dos percentuais foi obtida pelo total de queixas referidas); seção (F) - consumo de medicamentos alopáticos nos últimos 30 dias (sim ou não), consumo de plantas medicinais nos últimos 30 dias (sim ou não); uso combinado de medicamentos alopáticos e plantas medicinais no último mês (sim ou não).

\section{Atipicidade da coleta de dados}

O deslocamento da equipe até as comunidades ribeirinhas foi realizado por meio de embarcações particulares, ou por parcerias firmadas com uma organização não governamental (ONG) e pela SEMSA. A exceção foi a comunidade Nossa Senhora do Perpétuo Socorro do Itapéua, cujo acesso é feito por via terrestre. As viagens para coleta de dados nas comunidades próximas ao município tiveram a duração de um a três dias e foram realizadas aos finais de semana e feriados, exceto aquelas que duraram até 9 dias - período de recesso acadêmico.

O período em que foi desenvolvida a coleta de dados (cheia do rio Solimões), viabilizou a navegação até as comunidades. Foram navegados cerca de $2.000 \mathrm{~km}$ entre rios e lagos na zona rural de Coari, durante esta fase do SAMARA. Em consequência do período das cheias, a maioria das comunidades se encontrava inundada. Desse modo, o deslocamento do barco de apoio (balsas ou embarcações de maior porte) para a maioria das residências foi realizado com auxílio de voadeira (botes de alumínio com motor de popa). Os custos com a pesquisa foram elevados. Em comparação ao deslocamento terrestre com veículo automotivo, o custo com combustíveis representa cerca de sete vezes mais por causa do tipo de embarcação (barco de alumínio e motor 40hp). Além disso, o tempo de deslocamento foi grande e sujeito a variações climáticas, aspectos que envolveram itens como alimentação e estadia, que também impactaram nos custos.

Para melhor desempenho da equipe, os entrevistadores seguiram a estratégia adotada no treinamento, aplicando os questionários e realizando as medidas de antropometria e pressão arterial em pares. Esse aspecto contribuiu na redução do tempo médio de coleta de 60 minutos para 45 minutos por indivíduo. Após as entrevistas, foram realizadas consultas médicas e de enfermagem com os profissionais de saúde da equipe de pesquisa e dos respectivos órgãos (ONG e SEMSA), no barco de apoio.

\section{Georreferenciamento das comunidades}

As comunidades foram mapeadas por meio de um localizador GPS (Garmin eTrex 30, Olathe, Estados Unidos), com precisão de 3,6 metros (margem de erro da posição exata). Adotou-se o sistema de coordenadas geográficas (Lat Long) e o DATUM WGS84. Para determinar a distância das comunidades até a zona urbana do município, nas comunidades, utilizaram-se como ponto de referência as igrejas ou centros comunitários, e, na zona urbana, a referência foi a UBS que atende a população ribeirinha. Admitiu-se como distância percorrida o trajeto percorrido pelos rios entre os dois pontos de referência. Após a etapa de coleta de dados, as coordenadas foram transferidas para computador com auxílio do programa GPS TrackMaker, versão 13.9 (https://www.download-genius.com/en/download-k:gps-trackmaker-pro.html?aff.id=6499), e foram elaborados mapas.

\section{Aspectos éticos}

O estudo SAMARA - Médio Solimões foi aprovado pelo Comitê de Ética em Pesquisa da Escola de Enfermagem da Universidade de São Paulo sob no33560914.0.0000.5392. Os termos de Consentimento 
Livre e Esclarecido (TCLE) foram disponibilizados e aos que informaram dificuldades de leitura, os termos foram lidos pelos pesquisadores. Para assinatura, foram disponibilizados tinteiros com almofadas para colher as digitais dos que não sabiam assinar.

\section{Análise dos dados}

As análises foram realizadas por estatística descritiva, conforme as características de distribuição das variáveis. Os dados foram analisados por meio do programa IBM SPSS 20.0 for Windows (IBM Corp., Armonk, Estados Unidos).

\section{Resultados}

Foram entrevistados 492 sujeitos em 277 domicílios, nas 24 comunidades ribeirinhas selecionadas. Dentre os ribeirinhos entrevistados, a maioria é do sexo feminino (53\%), adultos entre 18-59 anos (88,6\%), com até 9 anos de estudos (68,5\%), apresentam como atividade econômica a pesca e a agricultura $(63,2 \%)$ e renda familiar mensal per capita de $\mathrm{R} \$ 241,60$, equivalente a $1 / 3$ do salário mínimo vigente. A maioria dos ribeirinhos relatou algum problema de saúde nos últimos 30 dias (77,4\%). Em relação aos cuidados com a saúde, a maioria utilizou medicamentos alopáticos nos últimos 30 dias (70,3\%) e 44,3\% utilizaram plantas medicinais (Tabela 3).

A maioria das comunidades não possui telefone público em funcionamento (87,5\%), não tem energia elétrica fornecida pela distribuidora local (75\%), e $83,3 \%$ não possui transporte (lancha) para uso comunitário (dados não apresentados em tabela).

Na Tabela 4, observa-se a distribuição dos sujeitos segundo as características das habitações. A disponibilidade de energia elétrica contínua (fornecedora de energia local) nas residências é limitada, e o uso de geradores movidos a gasolina ou diesel é frequente (72,6\%). Metade das casas tem de 1 a 3 cômodos e a água para consumo é adquirida diretamente dos rios e lagos (64,7\%), cujo tratamento mais comumente utilizado é hipoclorito $(72,2 \%)$. Quanto ao acesso ao município, cerca da metade $(51,4 \%)$ dos ribeirinhos navega menos de 50km (média 60,4km), e 51\% leva de 1-4 horas (média 4,2 horas) no deslocamento para acessar a zona urbana do município. A frequência com que visitam a sede municipal para $62,2 \%$ do grupo foi mensal $(62,2 \%)$ (Tabela 4).

O principal problema de saúde autorrelatado foi dor em geral $(45,2 \%)$. Dentre as dores, destacaram-se dor de cabeça $(56,6 \%)$, costas $(32,2 \%)$, musculares (4\%), articulações (3,6\%) e abdominais $(3,6 \%)$. Gripe $(7,5 \%)$ e sintomas relacionados como febre $(3,8 \%)$ e tosse $(0,9 \%)$ totalizaram $12,2 \%$. Doenças crônicas como hipertensão arterial $(9,5 \%)$, diabetes $(1,1 \%)$ e reumatismo $(0,4 \%)$ somaram $11 \%$. Dentre os problemas gastrointestinais $(8,9 \%)$, destacaram-se a diarreia $(32,7 \%)$, gastrite $(28,6 \%)$, azia $(22,4 \%)$ e vômito $(16,3 \%)$. Foram registradas infecções do trato urinário (6,7\%), anemias $(6,6 \%)$ e inflamações $(6,4 \%)$. Outros problemas de saúde autorrelatados (osteoporoses e verminoses) totalizaram 3\% (dados não apresentados em tabela).

\section{Discussão}

Considerando a incipiência de informações sistemáticas da população ribeirinha no Amazonas e a carência de políticas públicas específicas, o estudo SAMARA apontou diferentes aspectos que ilustram condições socioeconômicas, demográficas e de saúde deficitárias e que podem impactar de maneira negativa nas condições de vida dos ribeirinhos.

De modo geral, a população ribeirinha de Coari é jovem, apresenta baixo nível econômico e desenvolve atividades laborais voltadas à agricultura e pesca. As condições de moradia são deficitárias, sobretudo por carecer de saneamento básico. $\mathrm{O}$ acesso à energia e o tratamento da água são limitados e o acesso à zona urbana é restrito, levando os moradores a buscarem solucionar seus problemas de saúde na própria comunidade, especialmente pelo uso de medicamentos alopáticos. Os problemas de saúde são diversos, prevalecendo o autorrelato de problemas álgicos. 


\section{Tabela 3}

Características demográficas, socioeconômicas e de condições de saúde autorrelatadas dos ribeirinhos. Coari, Amazonas, Brasil, 2015.

\begin{tabular}{|c|c|c|}
\hline \multirow[t]{2}{*}{ Variáveis } & \multicolumn{2}{|c|}{$\begin{array}{l}\text { Ribeirinhos } \\
(\mathrm{N}=492)\end{array}$} \\
\hline & $\mathbf{n}$ & $\%$ \\
\hline \multicolumn{3}{|l|}{ Sexo } \\
\hline Feminino & 261 & 53,0 \\
\hline Masculino & 231 & 47,0 \\
\hline \multicolumn{3}{|l|}{ Idade (anos) } \\
\hline $18-39$ & 287 & 58,3 \\
\hline $40-59$ & 149 & 30,3 \\
\hline$\geq 60$ & 56 & 11,4 \\
\hline \multicolumn{3}{|l|}{ Raça } \\
\hline Parda & 438 & 89,0 \\
\hline Branca & 28 & 5,7 \\
\hline Negra & 14 & 2,9 \\
\hline Amarela & 6 & 1,2 \\
\hline Indígena & 6 & 1,2 \\
\hline \multicolumn{3}{|l|}{ Religião } \\
\hline Católico & 322 & 65,4 \\
\hline Evangélico & 153 & 31,1 \\
\hline Outras & 17 & 3,5 \\
\hline \multicolumn{3}{|l|}{ Situação conjugal } \\
\hline Vive só & 94 & 19,1 \\
\hline Vive acompanhado & 398 & 80,9 \\
\hline \multicolumn{3}{|l|}{ Escolaridade (anos) } \\
\hline Analfabeto & 48 & 9,7 \\
\hline $1-4$ & 146 & 29,7 \\
\hline $5-9$ & 143 & 29,1 \\
\hline$\geq 10$ & 155 & 31,5 \\
\hline \multicolumn{3}{|l|}{ Atividade laboral } \\
\hline Não exerce atividade laboral & 103 & 20,9 \\
\hline Agricultura e pesca & 311 & 63,2 \\
\hline Funcionário público * & 59 & 12,0 \\
\hline Outras & 19 & 3,9 \\
\hline \multicolumn{3}{|c|}{ Renda familiar mensal per capita em Reais (salário mínimos) ** } \\
\hline $10-85$ & 119 & 24,2 \\
\hline $86-140$ & 110 & 22,4 \\
\hline $141-240$ & 126 & 25,6 \\
\hline 241 ou mais & 137 & 27,8 \\
\hline \multicolumn{3}{|c|}{ Número de moradores no domicílio } \\
\hline $1-5$ & 246 & 50,0 \\
\hline$\geq 6$ & 246 & 50,0 \\
\hline \multicolumn{3}{|l|}{ Problema de saúde } \\
\hline Sim & 381 & 77,4 \\
\hline Não & 111 & 22,6 \\
\hline \multicolumn{3}{|l|}{ Uso de bebida alcoólica } \\
\hline Sim & 147 & 29,9 \\
\hline Não & 345 & 70,1 \\
\hline
\end{tabular}

(continua) 
Tabela 3 (continuação)

\begin{tabular}{|c|c|c|}
\hline \multirow[t]{2}{*}{ Variáveis } & \multicolumn{2}{|c|}{$\begin{array}{l}\text { Ribeirinhos } \\
(\mathrm{N}=492)\end{array}$} \\
\hline & $\mathbf{n}$ & $\%$ \\
\hline \multicolumn{3}{|l|}{ Fuma } \\
\hline Sim & 164 & 33,6 \\
\hline Não & 328 & 66,7 \\
\hline \multicolumn{3}{|c|}{ Procurou serviço de saúde no último mês } \\
\hline Sim & 124 & 25,2 \\
\hline Não & 368 & 74,8 \\
\hline \multicolumn{3}{|c|}{ Uso de medicamentos alopáticos no último mês } \\
\hline Sim & 346 & 70,3 \\
\hline Não & 146 & 29,7 \\
\hline \multicolumn{3}{|c|}{ Consumo de plantas medicinais no último mês } \\
\hline Sim & 218 & 44,3 \\
\hline Não & 274 & 55,7 \\
\hline \multicolumn{3}{|c|}{ Uso combinado de medicamentos alopáticos e plantas medicinais no último mês } \\
\hline Sim & 166 & 33,7 \\
\hline Não & 326 & 66,3 \\
\hline
\end{tabular}

* Professor, agente comunitário de saúde e merendeira;

** Referente a abril de 2015 ( $R \$ 788,00)$.

A taxa de analfabetismo $(9,7 \%)$ pode ser considerada alta, particularmente se comparada a estudos prévios conduzidos com ribeirinhos $(0,6 \%) 8$ e com as taxas identificadas nas principais capitais da Região Norte - Manaus (3,8\%) e Belém (3,3\%) 19. Embora nos últimos anos tenham ocorrido investimentos na formação de professores, criação de novas escolas e transporte escolar fluvial, o grupo analisado compreende adultos e idosos (18 a 92 anos) que talvez não tenham sido alcançados por essas melhorias. Eles, provavelmente, conviveram com a inexistência de escolas em algumas comunidades ou com a impossibilidade de se deslocar para outra de referência, por não haver transporte escolar. A formação contínua de licenciados para atuar no ensino básico no município ocorreu a partir do ano de 2010, em uma universidade pública no município. É possível que esse novo panorama tenha proporcionado a qualificação e a fixação dos professores nas comunidades e a expansão do ensino médio (1o a 3 o ano) nessas localidades, outrora limitado 7 .

A renda familiar mensal per capita dos ribeirinhos é baixa, aproximadamente 30\% do salário mínimo, contrastando com a zona urbana de Coari (130\% do salário mínimo), e da zona rural da cidade de Manaus (123\% do salário mínimo referente a 2015) 6. Investigação realizada com 340 ribeirinhos da mesma região apontou renda familiar per capita de $24 \%$ do valor do salário mínimo 8 , indicando que, embora tenha ocorrido um acréscimo na renda, talvez pela implementação de programas sociais, as condições econômicas ainda são limitadas 20. Isso se contrapõe ao constante aumento do PIB do município nas últimas décadas, e com o Índice de Desenvolvimento Humano (IDH) $(0,586) 13$, semelhante ao de países africanos subdesenvolvidos, como Zâmbia (0,586), Guiné Equatorial $(0,587)$ e Gana $(0,579) 21$. Essas evidências apontam sérias desigualdades no município, sobretudo entre zona rural e urbana.

Ainda que as condições socioeconômicas dos ribeirinhos sejam limitadas, a fartura de alimentos extraídos da floresta e bacias hidrográficas extensas parece suprir as condições mínimas para habitar a região, no entanto limita a melhoria de suas condições de vida. Esse panorama é atenuado pelo ambiente, cujas variações sazonais influenciam o modo de vida, levando à necessidade de os ribeirinhos se adaptarem para sobreviver 22. Dentre esses aspectos, o estudo apontou que os indivíduos percorrem em média 60,4km e demoram cerca de 4,2 horas para acessar a zona urbana, onde têm acesso aos serviços de saúde e diversos insumos para manutenção de suas famílias nas comunidades. 


\section{Tabela 4}

Distribuição dos sujeitos segundo as características da habitação e acesso à zona urbana do município. Coari, Amazonas Brasil, 2015.

\begin{tabular}{|c|c|c|}
\hline \multirow[t]{2}{*}{ Variáveis } & \multicolumn{2}{|c|}{$\begin{array}{l}\text { Ribeirinhos } \\
(\mathrm{N}=492)\end{array}$} \\
\hline & $\mathbf{n}$ & $\%$ \\
\hline \multicolumn{3}{|l|}{ Procedência da energia elétrica } \\
\hline Fornecedora de energia local & 135 & 27,4 \\
\hline Gerador de energia & 357 & 72,6 \\
\hline \multicolumn{3}{|l|}{ Número de cômodos por residência } \\
\hline $1-3$ & 247 & 50,2 \\
\hline 4 ou mais & 245 & 49,8 \\
\hline \multicolumn{3}{|c|}{ Procedência da água para consumo } \\
\hline Rio ou lago & 211 & 64,7 \\
\hline Poço & 127 & 25,8 \\
\hline Chuva & 47 & 9,5 \\
\hline Tratamento da água * & 355 & 72,2 \\
\hline Rádio & 294 & 59,8 \\
\hline Televisão & 363 & 73,8 \\
\hline Celular & 239 & 48,6 \\
\hline \multicolumn{3}{|c|}{ Distância da comunidade à zona urbana (km) } \\
\hline$<50$ & 253 & 51,4 \\
\hline $50-100$ & 139 & 28,3 \\
\hline$>100$ & 100 & 20,3 \\
\hline \multicolumn{3}{|c|}{ Tempo de deslocamento da comunidade à zona urbana (hora) } \\
\hline$<1$ & 54 & 11,0 \\
\hline $1-4$ & 251 & 51,0 \\
\hline$>4$ & 187 & 38,0 \\
\hline \multicolumn{3}{|l|}{ Frequência de idas à zona urbana } \\
\hline Mais de uma vez por mês ** & 151 & 30,7 \\
\hline Apenas uma vez por mês & 306 & 62,2 \\
\hline Não costumo ir & 35 & 7,1 \\
\hline
\end{tabular}

* Uso de hipoclorito;

** Todos os dias; até 3 vezes por semana; 1 vez por semana; 1 vez por quinzena.

Por mais que esses achados sejam expressivos, as limitações de acesso à zona urbana são menores nos períodos de cheias. As "rabetas" permitem que os ribeirinhos elaborem rotas alternativas e percorram em linha reta canais em meio à floresta alagada (igapó). Por outro lado, nesse período, não é possível desenvolver atividades agrícolas, e a invasão da água gera a necessidade de suspensão do piso das casas com tábuas "marombas". Quando as residências ficam submersas, seus habitantes são obrigados a deixá-las, dirigindo-se para a casa de familiares ou para a zona urbana, o que parece estar contribuindo para a migração de habitantes da zona rural para áreas periféricas da zona urbana no município. Entre agosto e outubro, há escassez de chuvas, quando os rios atingem seus menores níveis no ano, o que dificulta a navegação e isola geograficamente algumas comunidades. Entre novembro e abril, ocorre lentamente a elevação do nível dos rios e, a partir de maio, os níveis se elevam até alcançar seu pico máximo em julho.

Ao considerar o emaranhado de canais na floresta alagada (igapó, rios e lagos) durante o período das cheias, o georreferenciamento das comunidades e rotas, neste estudo, viabilizou a localização e trajeto. Adicionalmente, contribuiu para manutenção de informações geográficas que permitem verificar a distribuição das doenças, auxiliar na investigação das condições de acesso aos serviços de saúde, na 
elaboração de mapas com maior precisão, na replicação do estudo e elaboração de novas investigações com recortes diferentes, tais como estudos de seguimento. Além disso, é um importante instrumento para os gestores na articulação e planejamento de estratégias de cobertura dos serviços, possibilitando calcular os custos efetivos de atividades de saúde a serem desenvolvidas nas comunidades.

As limitações de acesso à zona urbana, atreladas à realidade econômica desfavorável, parecem impactar na frequência com que os ribeirinhos costumam ir ao município, em virtude da necessidade de aquisição de combustível para impulsionar as embarcações. Dessa forma, a maioria dos sujeitos se dirige até a sede municipal nas ocasiões em que recebem incentivos sociais como o bolsa família, aproveitando para vender seus produtos e adquirir alimentos básicos, insumos de saúde, dentre outros produtos. Ainda assim, os resultados indicaram que o consumo de medicamentos alopáticos nas comunidades é elevado, superando o uso de plantas medicinais, mesmo que as plantas sejam uma alternativa de fácil acesso e de baixo custo a essas populações 23. Parece que o uso de medicamentos alopáticos constitui uma alternativa aos serviços de saúde para essa população. Embora os efeitos dos medicamentos sejam amplamente reconhecidos, seu uso inapropriado pode ocasionar problemas à saúde 24. Tais aspectos, adicionados a deficiências educacionais, limitações de acesso à informação e uso conjunto com plantas medicinais, constituem um cenário com riscos potenciais à saúde. A terapia com o uso de plantas medicinais, aparentemente inofensiva, por ser "natural", pode levar à interação com medicamentos alopáticos 25 . Além disso, muitas plantas que têm um extrato biologicamente ativo perdem suas características ao serem manipuladas 26 .

A identificação de problemas de saúde autorreferidos possibilita o reconhecimento das condições de saúde de populações específicas em estudos populacionais 27 . Nessa perspectiva, sintomas álgicos das mais variadas origens foram os principais problemas de saúde autorrelatados. Dependendo da intensidade da dor (aguda ou crônica), esse quadro pode constituir importante razão para procura dos serviços de saúde 28. O quadro elevado desse sintoma talvez seja explicado pela atividade laboral exercida pela maioria dos ribeirinhos que vivem de agricultura e pesca, o que requer uso excessivo de força física e sobrecarga de peso, possivelmente desenvolvidas sem qualquer cuidado com a ergonomia. Portanto, o primeiro recurso para alívio imediato da dor é o uso de medicamentos alopáticos.

As doenças crônicas, sobretudo a hipertensão arterial autorreferida, foram pouco relatadas quando contrastada com estudo realizado com ribeirinhos no rio Madeira (Rondônia), cuja prevalência foi de $26 \% 29$. Contudo, o autorrelato sofre o viés de mensuração, uma vez que essa população tem baixo acesso ao diagnóstico clínico. Além disso, na maioria das comunidades não havia aparelhos para mensuração dos níveis pressóricos.

Problemas do sistema gastrointestinal também foram frequentes. As condições precárias de saneamento, com o consumo de água imprópria diretamente dos rios e lagos, podem contribuir para seu incremento, com o advento de doenças de veiculação hídrica, tais como as enteroparasitoses 30 .

Embora a malária represente uma das principais doenças infecciosas na região amazônica, não foram registrados relatos nos últimos 30 dias. Esse aspecto talvez seja explicado pelo período de coleta de dados que ocorreu durante as cheias, quando o ambiente é menos favorável à proliferação do vetor transmissor da doença 31 e por consequência à ocorrência de casos 32 .

Dentre os aspectos metodológicos, é importante destacar as dificuldades no sorteio das residências. Isso ocorre porque não há um padrão de distribuição das residências nas comunidades. Ademais, existem casas dispersas ao longo das margens dos rios, dificultando o acesso às residências e ao sorteio, sobretudo em regiões de várzeas nos períodos de cheia. Outra investigação realizada com ribeirinhos (rio Madeira) em Porto Velho apontou dificuldades no processo de amostragem, o que levou à realização de um censo. No rio Madeira, foram investigadas 15 comunidades ribeirinhas, 14 delas localizadas próximas a uma cachoeira, viabilizando o acesso e a possibilidade de realizar o censo 29. Por outro lado, quando as áreas territoriais nos dois estudos são comparadas, Município de Porto Velho e áreas adjacentes $\left(34.082 \mathrm{~km}^{2}\right)$, isso representa cerca da metade da área do Município de Coari $\left(57.000 \mathrm{~km}^{2}\right)$, por isso, neste inquérito, não foi possível a realização de um censo. Outro aspecto é a heterogeneidade das comunidades e suas populações (acesso à zona urbana, recursos estruturais das comunidades, entre outros) entre as regiões de lagos e rios da zona rural de Coari.

Ainda entre os aspectos metodológicos, a forma de tabulação dos dados do SIAB para a população rural a respeito da idade é apresentada em intervalos, dentre eles o de 15-19 anos, o que limitou a identificação com exatidão da população de referência do estudo. Nesse sentido, assumiu-se a popula- 
ção desse intervalo como a de referência para a população de 18 e 19 anos. Essa decisão ocorreu, também, por coerência com a proposta inicial encaminhada ao Comitê de Ética em Pesquisa. Assim, os pesquisadores entenderam que, por se tratar de uma estimativa do quantitativo da população total de referência, para fins de correção do número amostral por população finita, optou-se por realizar um cálculo amostral com uma população maior (quantidade de indivíduos do intervalo de 15-19 anos), do que errar assumindo um quantitativo menor que o real. Outro aspecto a ser levado em conta é que, para o tamanho final da amostra, foram considerados inicialmente $20 \%$ de perdas que não ocorreram na prática, pois foi utilizada estratégia de reposição. De mais a mais, apesar do quantitativo estimado da amostra de 470 sujeitos ( 374 acrescidos da proporção de perdas e efeito do desenho), ao final da coleta de dados em todas as comunidades, foram contabilizados 492 indivíduos entrevistados, obedecendo ao rigor do sorteio e seleção das residências. Além do mais, o número expressivo de indivíduos alcançados frente ao estimado inicialmente, confere maior poder ao estudo, a despeito do problema inicial de falta de correto intervalo populacional de referência.

O estudo apresentou limitações no que concerne ao uso dos dados demográficos utilizados para cálculo amostral, por representarem apenas a população coberta pelo sistema de saúde. Todavia, no âmbito local, compreenderam as informações disponíveis mais abrangentes e confiáveis. Chuvas torrenciais no período, precária manutenção das embarcações, obstrução dos canais de lagos e igapós por densa vegetação aquática dificultaram as atividades de campo. Porém, não foram impeditivas para a coleta de dados conforme o planejado. Ressalta-se que o estudo traz informações de condições de saúde dessa população em época de cheia; é possível que, em tempo de seca, algumas situações sejam diferentes, o que necessitaria de estudos futuros.

\section{Conclusão}

O estudo apontou baixo nível econômico entre os ribeirinhos. O acesso ao município é limitado e os problemas de saúde são, sobretudo, queixas álgicas, solucionadas na própria comunidade, geralmente com o uso de medicamentos alopáticos. As condições econômicas desfavoráveis, aliadas ao ambiente em constante mudança e limitações geográficas, constituem importantes barreiras para o acesso aos serviços de saúde e à melhoria das condições de vida dos ribeirinhos.

O ambiente atípico, com densa floresta tropical e rios colossais, chuvas torrenciais, também limita a aquisição de informações de caráter científico por pesquisadores. Nesse aspecto, o desenvolvimento de estudos no ambiente amazônico, exige suporte de recurso financeiro e esforço adicional em relação aos desenvolvidos em áreas urbanas. Dessa maneira, embora neste estudo sejam pontuados apenas os aspectos metodológicos e alguns resultados, espera-se que esses achados possam contribuir no desenvolvimento de futuras investigações entre os povos ribeirinhos da Amazônia e balizar aspectos que permitam a articulação de políticas públicas a esses povos.

\section{Colaboradores}

A. S. M. Gama e T. G. Fernandes contribuíram na concepção do trabalho, aquisição, análise e interpretação de dados, redação e revisão crítica do conteúdo. R. C. P. Parente contribuiu na concepção da amostragem e na revisão final da versão a ser publicada. S. R. Secoli contribuiu na concepção do trabalho, aquisição, análise e interpretação de dados; revisão crítica do conteúdo; e aprovação final da versão a ser publicada.

\section{Agradecimentos}

Fundação de Amparo à Pesquisa do Estado do Amazonas. 


\section{Referências}

1. Bezerra VM, Medeiros DS, Gomes KO, Souzas R, Giatti L, Steffens AP, et al. Health survey in Quilombola communities (descendants of Afro-Brazilian slaves who escaped from slave plantations that existed in Brazil until abolition in 1888) in Vitória da Conquista in the state of Bahia (COMQUISTA Project), Brazil: methodological aspects and descriptive analysis. Ciênc Saúde Coletiva 2014; 19:1835-47.

2. Viacava F. Informações em saúde: a importância dos inquéritos populacionais. Ciênc Saúde Coletiva 2002; 7:607-21.

3. Malta DC, Leal MC, Costa MFL, Morais Neto OL. Inquéritos Nacionais de Saúde: experiência acumulada e proposta para o inquérito de saúde brasileiro. Rev Bras Epidemiol 2008; 11 Suppl 1:159-67.

4. Fraxe TJP, Pereira HS, Witkoski AC. Comunidades ribeirinhas amazônicas: modos de vida e uso dos recursos naturais. Manaus: EDUA; 2007.

5. Instituto Brasileiro de Geografia e Estatística. Cadastro de municípios localizados na Amazônia Legal. http://www.ibge.gov.br/ho me/geociencias/geografia/amazonialegal.sht $\mathrm{m} ? \mathrm{c}=2$ (acessado em 05/Jun/2016).

6. Instituto Brasileiro de Geografia e Estatística. Sinopse do censo demográfico de 2010. http://www.censo2010.ibge.gov.br/sinopse/ index.php?dados $=10 \& u f=00$ (acessado em 05/ Jun/2016).

7. Sousa IS. As condições de vida e saneamento nas comunidades da área de influência do gasoduto Coari-Manaus em Manacapuru - AM. Hygeia (Uberlândia) 2009; 5:88-98.

8. Moretti-Pires RO, Corradi-Webster CM. Adaptação e validação do Alcohol Use Disorder Identification Test (AUDIT) para população ribeirinha do interior da Amazônia, Brasil. Cad Saúde Pública 2011; 27:497-509.

9. Mendes LSA, Pontes FAR, Silva SSC, BucherMaluschke JSNF, Reis DC, Silva SDB. The ecological engagement in the context of an Amazon river Village. Interam J Psychol 2008; 42:1-10.

10. Hacon SS, Dórea JG, Fonseca MF, Oliveira BA, Mourão DS, Ruiz CM, et al. The Influence of changes in lifestyle and mercury exposure in riverine populations of the Madeira River (Amazon Basin) near a hydroelectric project. Int J Environ Res Public Health 2014; 1 1:2437-55.

11. Adami YL, Rodrigues G, Alves MC, Moraes MAP, Banic DM, Maia-Herzog M. New records of Mansonella ozzardi: a parasite that is spreading from the state of Amazonas to previously uninfected areas of the state of acre in the Purus River region. Mem Inst Oswaldo Cruz 2014; 109:87-92.

12. Katsuragawa TH, Gil LHS, Tada MS, Silva LHC. Endemic and epidemic diseases in Amazonia. Estud Av 2008; 22:111-41.
13. Instituto Brasileiro de Geografia e Estatísti ca. Cidades: PIB per capita a preços correntes - 2013 http://cidades.ibge.gov.br/compara mun/compara.php?lang $=\& \operatorname{coduf}=13 \&$ idte $\mathrm{ma}=16 \& \operatorname{codv}=\mathrm{v} 07 \&$ search $=$ amazonas $\mid$ coari $\mid$ sintese-das-informacoes-\# (acessado em 16/ Mai/2016).

14. Secretaria de Atenção à Saúde, Ministério da Saúde. Cadastro Nacional de Estabelecimentos de Saúde (CNES) 2016. Estabelecimento de saúde do município: Coari. http://cnes2. datasus.gov.br/Lista_Es_Municipio.asp? VEstado $=13 \&$ VCodMunicipio $=130120 \&$ NomeEstado=AMAZONAS (acessado em 07/ Jun/2016)

15. Matsudo S, Araújo T, Matsudo V, Andrade D, Andrade E, Oliveira LC, et al. Questionário internacional de atividade física (IPAQ): estudo de validade e reprodutibilidade no Brasil. Rev Bras Ativ Fís Saúde 2001; 6:5-18.

16. Craig CL, Marshall AL, Sjöström M, Bauman AE, Booth ML, Ainsworth BE, et al. International physical activity questionnaire: 12 -country reliability and validity. Med Sci Sports Exerc 2003; 35:1381-95.

17. Nunes DP, Duarte YA, Santos JL, Lebrão ML. Screening for frailty in older adults using a self-reported instrument. Rev Saúde Pública 2015; 49:2.

18. Sociedade Brasileira de Cardiologia; Sociedade Brasileira de Hipertensão; Sociedade Brasileira de Nefrologia. VI diretrizes brasileiras de hipertensão. Arq Bras Cardiol 2010; 95(1 Suppl 1):1-51.

19. Instituto Brasileiro de Geografia e Estatística. Indicadores sociais e municipais. http://www. ibge.gov.br/home/estatistica/populacao/cen so2010/indicadores_sociais_municipais/tabe las_pdf/tab28.pdf (acessado em 05/Jun/2016).

20. Piperata BA, Spence JE, Da-Gloria P, Hubbe $M$. The nutrition transition in amazonia: rapid economic change and its impact on growth and development in Ribeirinhos. Am J Phys Anthropol 2011; 146:1-13.

21. Programa das Nações Unidas para o Desenvolvimento. Ranking IDH Global 2014. http:// www.br.undp.org/content/brazil/pt/home/ idh0/rankings/idh-global.html (acessado em 05/Jun/2016).

22. Harris M. The rhythm of life on the amazon floodplain: seasonality and sociality in a riverine village. J R Anthropol Inst 1998; 4:65-82.

23. Haeffner R, Heck RM, Ceolin T, Jardim VMR, Barbieri RL. Plantas medicinais utilizadas para o alívio da dor pelos agricultores ecológicos do Sul do Brasil. Rev Eletrônica Enferm 2012; 14:596-602.

24. Walckiers D, Van der Heyden J, Tafforeau J. Factors associated with excessive polypharmacy in older people. Arch Public Health 2015; 73:50. 
25. Gohil KJ, Patel JA. Herbdrug interactions: a review and study based on assessment of clinical case reports in literature. Indian J Pharmacol 2007; 39:129-39.

26. Villas Bôas GK, Gadelha CA. Oportunidades na indústria de medicamentos e a lógica do desenvolvimento local baseado nos biomas brasileiros: bases para a discussão de uma política nacional. Cad Saúde Pública 2007; 23:1463-71.

27. Francisco PM, Barros MB, Segri NJ, Alves MC. Comparison of estimates of population-based surveys. Rev Saúde Pública 2013; 47:60-8.

28. Ferreira GD, Silva MC, Rombaldi AJ, Wrege ED, Siqueira FV, Hallal PC. Prevalence and associated factors of back pain in adults from southern Brazil: a population-based study. Braz J Phys Ther (Impr.) 2011; 15:31-6.

29. Oliveira BFA, Mourão DS, Gomes N, Costa JMC, Souza AV, Bastos WR, et al. Prevalência de hipertensão arterial em comunidades ribeirinhas do Rio Madeira, Amazônia Ocidental Brasileira. Cad Saúde Pública 2013; 29:161730.
30. Silva AMB, Bouth RC, Costa KS, Carvalho DC, Hirai KE, Prado RR, et al. Occurrence of intestinal parasites in riverine communities in the City of Igarapé Miri, Pará State, Brazil. Rev Pan-Amazônica Saúde 2014; 5:45-51.

31. Barbosa LMC, Souto RNP, Ferreira RMA, Scarpassa VM. Composition, abundance and aspects of temporal variation in the distribution of Anopheles species in an area of Eastern Amazonia. Rev Soc Bras Med Trop 2014; 47:313-20.

32. Monteiro MRCC, Fernandes SC, Ribeiro MC. Aspectos clínicos e epidemiológicos da malária em um hospital universitário de Belém, Estado do Pará, Brasil. Rev Pan-Amazônica Saúde 2013; 4:33-43. 


\section{Abstract}

Population-based health surveys are important tools for identifying disease determinants, especially in regions with widely dispersed populations and low health system coverage. The aim of this study was to describe the principal methodological aspects and to describe the socioeconomic, demographic, and health characteristics of the riverine populations of Coari, Amazonas State, Brazil. This was a population-based cross-sectional study in river-dwelling communities in the rural area of Coari, from April to July 2015. The probabilistic cluster sample consisted of 492 individuals. The results showed that the majority of the river-dwellers were females (53\%), had up to 9 years of schooling (68.5\%), and earned a monthly family income equivalent to one-third the minimum wage. The health problems reported in the previous 30 days featured conditions involving pain (45.2\%). The main healthcare resources were allopathic medicines (70.3\%), exceeding herbal remedies (44.3\%). The river-dwellers travel an average of $60.4 \mathrm{~km}$ and take some 4.2 hours to reach the urban area of Coari. The riverine population generally presents low economic status and limited access to the urban area. Health problems are mostly solved with allopathic medicines. Geographic characteristics, as barriers to access to health services and to improvements in living conditions for the riverine population, can limit the collection of epidemiological data on these populations.

Health Surveys; Health Status; Rural Population

\section{Resumen}

Las encuestas poblacionales de salud son instrumentos importantes para el reconocimiento de determinantes de morbilidades, sobre todo en regiones de gran dispersión demográfica y baja cobertura del sistema de salud. El objetivo de este estudio fue presentar los principales aspectos metodológicos y descibir las características socioeconómicas, demográficas y de salud los ribereños de Coari, Amazonas, Brasil. Se trata de un estudio transversal con base poblacional, realizado con ribereños residentes en la zona rural del municipio de Coari, durante el período de abril a julio de 2015. La muestra probabilística por conglomerados estaba compuesta por 492 sujetos. Los resultados indicaron que la mayoría de los ribereños es de sexo femenino (53,0\%), tiene hasta 9 años de estudios (68,5\%), presenta de media una renta familiar mensual equivalente a $1 / 3$ del salario mínimo. Entre los problemas de salud relatados en los últimos 30 días, se destacaron las quejas álgicas (45,2\%). Los principales recursos utilizados en los cuidados con la salud fueron medicamentos alopáticos (70,3\%), superando el uso de plantas medicinales (44,3\%). Los ribereños navegan de media $60,4 \mathrm{~km}$ y tardan casi 4,2 horas para acceder a la zona urbana del municipio. De manera general, la población ribereña estudiada está caracterizada por el bajo nivel económico y acceso limitado a zonas urbanas. Los problemas de salud se solucionan la mayor parte de las veces mediante el uso de medicamentos alopáticos. Las limitaciones geográficas que constituyen barreras al acceso a los servicios de salud, y a la mejoría de las condiciones de vida de los ribereños, pueden limitar la obtención de información epidemiológica de esa población.

Encuestas Epidemiológicas; Estado de Salud; Población Rural
Recebido em 09/Jan/2017

Versão final reapresentada em 18/Jun/2017 Aprovado em 05/Jul/2017 